УДК 51(477)

\author{
L. Bazylevych, I. Oleksiv, Ya. Prytula, M. Zarichnyi
}

\title{
IVAN PESIN (TO HIS 90TH ANNIVERSARY)
}

\begin{abstract}
L. Bazylevych, I. Oleksiv, Ya. Prytula, M. Zarichnyi. Ivan Pesin (to his 90th anniversary), Mat. Stud. 53 (2020), 221-224.

The note is devoted to biography and achievements of Ukrainian mathematician Ivan Pesin (1930-1993).
\end{abstract}

Ivan Pesin was born on January 7, 1930 in Turka, a small town (now in the Lviv region). He spent his childhood in his father's family in the ancient Ukrainian city of Kremenets. The hardest and darkest memories of his childhood were related to the three years of German occupation during World War II.

In 1947, after high school, he entered the Faculty of Physics and Mathematics of Lviv University. In his senior years, he became an active participant in a seminar led by professor L. Volkovyskii, the founder of modern school of complex analysis in Lviv.

In 1952, I. Pesin graduated with honors from the university and received a recommendation for admission to graduate school. Professor L. Volkovyskii became his scientific supervisor. During his postgraduate studies Pesin obtained important results in the theory of quasiconformal mappings. These results formed the basis of his $\mathrm{PhD}$ thesis "The Metric Properties of Quasiconformal Mappings", which he defended on Octo-

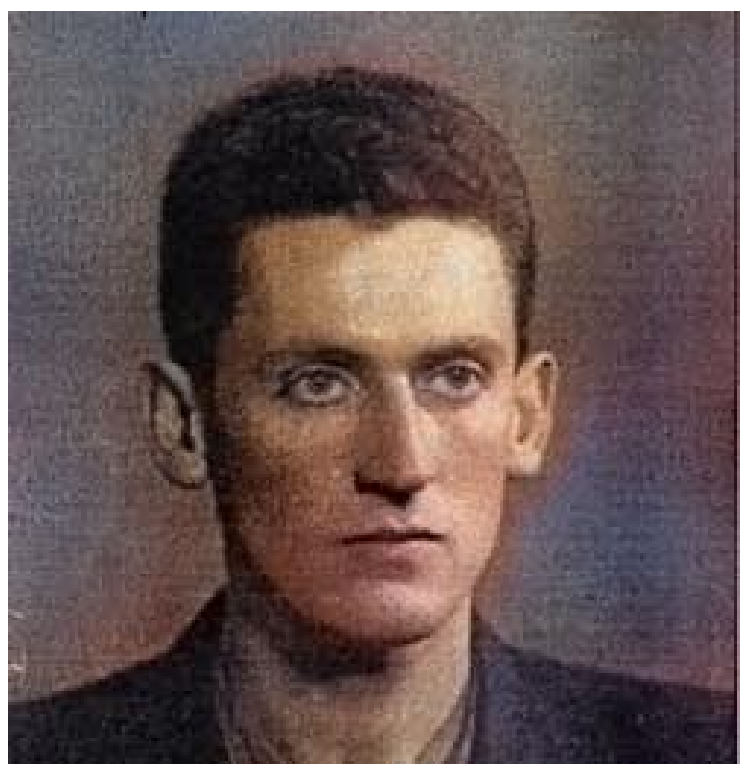
ber 24, 1955.

The results in the theory of quasiconformal mappings concerned extension of Lavrentiev's definition of quasiconformity. In $[11,12]$ Pesin replaced in the dilatation condition the family of concentric circles by a topological image of such a family not to much distorted. He worked with sense-preserving homeomorphisms of the unit disk onto itself and defined the class of generalized $Q$-quasiconformal mappings. Some important properties of this class were established in $[3,11,12]$ : every $Q$-quasiconformal mapping is absolutely continuous; the uniform limit of a sequence of $Q$-quasiconformal mappings is again a $Q$-quasiconformal mapping; some classes of null-sets in the sense of [1] are preserved by $Q$-quasiconformal mappings. Reshetnyak [14] used Pesin's approach to define the notion of quasiregularity in space [14]. See the survey article [6] for more information.

2020 Mathematics Subject Classification:01A70.

Keywords: Ivan Pesin; quasiconformal mapping; real analysis. doi:10.30970/ms.53.2.221-224

(C) L. Bazylevych, I. Oleksiv, Ya. Prytula, M. Zarichnyi, 2020 
Later, this class was extended by $\mathrm{P}$. Caraman to dimension $n \geq 2$ under the name of Markuševič-Pesin [4]. A significant part of Caraman's book [5] is devoted to proving of equivalence of and Gehring's metric definition and Markuševič-Pesin's one.

Note also that in [7] the property of removability in the class of conformal maps in space is established for closed sets located on smooth enough curves.

During his scientific activity, I. Pesin made a significant contribution not only to the development of the theory of quasiconformal mappings, but also to the theory of functions of a real variable and geometric topology. His broad scientific outlook, deep knowledge and non-standard thinking allowed him to formulate new interesting problems and propose approaches to solve them.

I. Pesin was always inspired by problems that could be formulated without using complex mathematical concepts, but the solutions of which were not at all obvious and could lead to unexpected conclusions. In connection to this, let us mention that in his note [12], Pesin provided an unexpectedly short proof that the length of the Cantor dust, i.e. the square $C \times C$ of the linear Cantor set $C$, is infinite. This proof definitely deserves to be placed in "The Book" (according to Paul Erdős in this book God keeps the most elegant proof of each mathematical theorem).

In [13], he gave a concise exposition of the Carathéodory theory of prime ends based on geometric and topological considerations.

When working with the Fréchet distance (some results of [2] concern this distance) he conjectured that one can consider also non-monotonic change of parameter in its definition. It was proved later that this actually leads to a new metric on the set of curves, and this metric founds applications in computer vision and pattern recognition.

The aim of the paper [8] was to attract the reader's attention to some problems that lie between the function theory and topology (the authors use the term "mixed problems"). Pesin was the author of the result from the first section of the paper, it concerned minimal isotopies of polygonal simple curves in plane. The section ended with the open problem whether two homotopic curves on a two-dimensional manifold are isotopic by means of an isotopy that "sweeps" the same region as the homotopy does. The connections between the function theory and topology were also emphasized in the paper [2].

In 1966, I. Pesin published the monograph "Development of the Notion of Integral" (in Russian). It contained, in modern notation and terminology, expositions of integration theories in their historical perspective. The book was clearly and accurately written. In 1970 its English translation was published under the title "Classical and Modern Integration Theories". Samuel Kots, the translator and editor, wrote that the book "is an insightful and well-motivated exposition of the classical theory of integration written by the distinguished Soviet mathematician Ivan N. Pesin, whose main contributions are in the field of quasiconformal transformations, Riemann surfaces, and the problems related to the theory of functions of real variable." In 2014 the 2 nd edition of the monograph was published [10].

There are 22 mathematical works (co)authored by I. Pesin.

In addition to his research activity, Pesin devoted a lot of his time and efforts to teaching mathematics. Numerous participants of his seminar (same sessions of it were held in Pesin's apartment) later became professional mathematicians. Pesin had five descendants (A. Kopylov, S. Ponomarev, I. Oleksiv, M. Brodovych, L. Bazylevych). 
He published an interesting paper on using quantifiers in university classes in a special didactics journal. In 1984, Pesin left his teaching job for health reasons.

Pesin was a man of wide culture, he read books in different languages and encouraged his students to do so. He had a rich library in his apartment, in mathematics but not only, and allowed his colleagues and students to borrow books from it. Fluent in different languages, Pesin often switched from one to another in conversation.

For three decades, I. Pesin conducted active scientific and pedagogical activity in the conditions of constant struggle against the incurable illness, which struck him, a twentyyear-old young man, in 1951. Because medicine was powerless in this case, he had to rely only on himself. In this struggle, which required constant exertion of physical and spiritual forces, he tried many different techniques and approaches, including physical exercise, sports (including sailing on the Hlynna Navariya lake), fasting. Physical infirmity was combined in him with the power of spirit.

I. Pesin wrote a series of handwritten psychological works in which he presented his observations, shared reflections and conclusions from many years of experience of life in the difficult conditions that fell to his lot.

I. Pesin's manuscripts are stored in the holdings of the Scientific Library of the University.

Ivan Pesin died on July 9, 1993.

\section{REFERENCES}

1. L. Ahlfors, A. Beur1ing, Conformal invariants and function-theoretic nullsets, Acta Math., 83 (1950), $100-129$.

2. L. E. Bazilevich, I. N. Pesin, Two problems concerning Fréchet distance and lines of evasion, Siberian Mathematical Journal, 22, 495-501(1981)

3. P.P. Belinskij and I.N. Pesin, On the closure of the class of differentiable quasiconformal mappings, Dokl. Akad. Nauk SSSR 102 (1955), 865-866 (in Russian).

4. P. Caraman, Homeomorfisme Cvasiconforme $n$-Dimensionale, Ed. Acad. R. S. R., Bucureşti (1968); $n$-Dimensional Quasiconformal Homeomorphisms, Ed. Acad. R. S. R., Bucureşti and Abacus Press, Tunbridge Wells, Kent (1974).

5. P. Caraman, $N$-dimensional Quasiconformal, QCf, Mappings. Front Cover. Petru Caraman. Ed. Acad., 1974 - 551 pages.

6. Andrean C. Cazacu, Foundations of quasiconformal mappings. Handbook of Complex Analysis, Geometric Function Theory, Elsevier B. V. 2, 687-755 (2005).

7. A. P. Kopylov, I. N. Pesin, Removability of certain sets in the class of three-dimensional quasiconformal mappings, Math. Notes, 7:6 (1970), 432-434.

8. I. Ya. Oleksiv, I. N. Pesin, Three problems on minimal isotopies and on approximation of curves and surfaces by homeomorphisms, Sibirskij matematiceskij zurnal (1977), Volume: 18, Issue: 5, 1140-1158.

9. I.N. Pesin, On the theory of generalized $Q$-quasiconformal mappings, Dokl. Akad. Nauk SSSR 102 (1955), 223-224 (in Russian).

10. Ivan N. Pesin, Classical and Modern Integration Theories, 1st Edition, Academic Press, 1970.; 2nd Edition, 2014, 218 pp.

11. I. N. Pesin, Metric properties of $Q$-quasiconformal mappings, Mat. Sb. (N.S.), 40(82):3 (1956), 281-294.

12. I. N. Pesin, On the lenghth of one everywhere discontinuous set of points, Uspekhi mat. nauk, 1955, V. 10, issue 3(65), 153 (In Russian) 
13. I. N. Pesin, Carathéodory theory of prime ends, Sibirskij matematiceskij zurnal (1966) Volume: 7, Issue: 5, page 1087-1094.

14. Yu.G. Reshetnyak, Space mappings with bounded distortion, Sibirsk. Mat. Zh. 8 (3) (1967), 629-658 (in Russian).

Faculty of Mechanics and Mathematics, Ivan Franko National University of Lviv,

1 Universytetska Street, 79000 Lviv, Ukraine

izar@litech.lviv.ua

Lviv Polytechnic National University,

12 Stepana Bandery Street, 79000 Lviv, Ukraine

ihor.oleksiv@gmail.com

Faculty of Mechanics and Mathematics, Ivan Franko National University of Lviv,

1 Universytetska Street,

79000 Lviv, Ukraine

ya.g.prytula@gmail.com

Faculty of Mechanics and Mathematics,

Ivan Franko National University of Lviv, 1 Universytetska Street,

79000 Lviv, Ukraine

zarichnyi@yahoo.com 